DrAFT, 20140722

Preprint typeset using $\mathrm{IAT}_{\mathrm{E}} \mathrm{X}$ style emulateapj v. 5/2/11

\title{
ANISOTROPIC LYMAN-ALPHA EMISSION
}

\author{
Zheng Zheng and Joshua Wallace \\ Department of Physics and Astronomy, University of Utah, 115 South 1400 East, Salt Lake City, UT 84112 \\ Draft, 20140722
}

\begin{abstract}
As a result of resonant scatterings off hydrogen atoms, Ly $\alpha$ emission from star-forming galaxies provides a probe of the (hardly isotropic) neutral gas environment around them. We study the effect of the environmental anisotropy on the observed Ly $\alpha$ emission by performing radiative transfer calculations for models of neutral hydrogen clouds with prescriptions of spatial and kinematic anisotropies. The environmental anisotropy leads to corresponding anisotropy in the Ly $\alpha$ flux and spectral properties and induces correlations among them. The Ly $\alpha$ flux (or observed luminosity) depends on the viewing angle and shows an approximate correlation with the initial Ly $\alpha$ optical depth in the viewing direction relative to those in all other directions. The distribution of Ly $\alpha$ flux from a set of randomly oriented clouds is skewed to high values, providing a natural contribution to the Ly $\alpha$ equivalent width (EW) distribution seen in observation. A narrower EW distribution is found at a larger peak offset of the Ly $\alpha$ line, similar to the trend suggested in observation. The peak offset appears to correlate with the line shape (full width at half maximum and asymmetry), pointing to a possibility of using Ly $\alpha$ line features alone to determine the systemic redshifts of galaxies. The study suggests that anisotropies in the spatial and kinematic distributions of neutral hydrogen can be an important ingredient in shaping the observed properties of Ly $\alpha$ emission from star-forming galaxies. We discuss the implications of using Ly $\alpha$ emission to probe the circumgalactic and intergalactic environments of galaxies.
\end{abstract}

Subject headings: cosmology: observations - galaxies: high-redshift — galaxies: statistics — intergalactic medium - radiative transfer - scattering

\section{INTRODUCTION}

Ly $\alpha$ emission from reprocessed ionizing photons in star-forming galaxies is a prominent feature that has been used to detect high redshift galaxies (e.g., Partridge \& Peebles 1967; Rhoads et al. 2003; Gawiser et al. 2007; Ouchi et al. 2008; Hill et al. 2008; Guaita et al. 2010; Ciardullo et al. 2012). Such Lya emitting galaxies (or Ly $\alpha$ emitters; LAEs) have become an important laboratory to study galaxy formation, large-scale structure, and cosmic reionization. The resonant scattering of Ly $\alpha$ photons with neutral hydrogen atoms in the circumgalactic and intergalactic media (CGM and IGM) also potentially opens a window to probe the spatial and kinematic environments of CGM and IGM from Ly $\alpha$ emission. In this paper, we perform a theoretical study of the effect of anisotropy in the environment on Ly $\alpha$ emission properties and study the implications of such an effect in using $\operatorname{Ly} \alpha$ emission to probe galaxy environment.

Anisotropic gas distributions are common in galaxies and their surrounding environments. The anisotropy can show up both spatially and kinematically. The gas distribution around star-forming regions (likely clumps on a galaxy disk) is already anisotropic. The galactic winds from star formation are ubiquitous and typically show a bipolar outflow pattern (e.g.. Bland \& Tullv 1988; Shopbell \& Bland-Hawthorn 1998; Veilleux \& Rupke 2002; Shapley et al. 2003; Weiner et al. 2009; Rubin et al. 2013). The cold gas being supplied for star formation could be accreted from streams and filaments (e.g., Kereš et al. 2005; Dekel et al. 2009). Even on the IGM scale, the density field and the velocity field still show appreciable fluc- tuations (e.g., Zheng et al. 2010). That is, spatial and kinematic anisotropies can exist in galactic environments on all scales. The resonant scatterings of Ly $\alpha$ photons would enable them to explore such anisotropies and encode information in the Ly $\alpha$ emission properties.

The anisotropic gas density and velocity distributions are naturally produced in hydrodynamic simulations of galaxy formation. Monte Carlo Ly $\alpha$ radiative transfer calculation has been performed for individual simulated galaxies. For example, Laursen et al. (2009) notice the anisotropic escape of $\operatorname{Ly} \alpha$ emission in nine simulated galaxies. Barnes et al. (2011) show that emerging Ly $\alpha$ spectra from three simulated galaxies vary strongly with the viewing angle. The escape fraction of Ly $\alpha$ emission in the simulated galaxies in Yajima et al. (2012) exhibits strong dependence on galaxy morphology and orientation, and for a disk galaxy the escaping $\operatorname{Ly} \alpha$ photons are confined to a direction perpendicular to the disk. Verhamme et al. (2012) also find that $\operatorname{Ly} \alpha$ properties strongly depend on the disk orientation in one simulated galaxy with an outflowing velocity field.

As a statistical study, Zheng et al. (2010) perform Lya radiative transfer calculation for about $2 \times 10^{5}$ sources in a radiative hydrodynamic cosmological reionization simulation. Given the resolution of the simulation, the density and velocity anisotropies come from gas in CGM and IGM. The Ly $\alpha$ emission properties (luminosity, surface brightness, and spectra) are found to depend on the viewing angle, as a result of the density and velocity distributions (environment). Because of the environment dependent radiative transfer, the Ly $\alpha$ emission properties show correlations among themselves, and new effects in the spatial clustering of LAEs are induced (Zheng et al. 2011a; also see Wyithe \& Dijkstra 2011; 
Behrens \& Niemeyer 2013).

On the analytic side, solutions are usually found for simple configurations of uniform media, e.g., static planeparallel uniform slabs (Harrington 1973; Neufeld 1990), static uniform spheres (Dijkstra et al. 2006), and uniform media experiencing Hubble expansion (in the diffusion regime; Loeb \& Rybicki 1999). Numerical solutions or simulations for analytical setups are also usually focused on uniform slabs (e.g., Auer 1968; Averv \& House 1968; Adams 1972; Ahn et al. 2000, 2001, 2002), or uniform spheres or isotropic systems (e.g., Loeb \& Rvbicki 1999; Ahn \& Lee 2002; Zheng \& Miralda-Escudé 2002; Diikstra et al. 2006; Verhamme et al. 2006; Rov et al. 2009, 2010).

Given the potential importance of the anisotropy in the observed properties of LAEs, it is useful to investigate Ly $\alpha$ emission with systems of analytic setups incorporating prescriptions of anisotropy. Such an investigation can guide our analyses of Ly $\alpha$ emission from simulated galaxies and help our understanding of the role of anisotropy in shaping the Ly $\alpha$ emission from LAEs. In this paper, we perform such a study.

The structure of the paper is as follows. In $\S[2$, we describe how we build models of neutral hydrogen clouds with spatial or kinematic anisotropy for Ly $\alpha$ radiative transfer calculation. Then we present the results of anisotropies in the escaping $\operatorname{Ly} \alpha$ emission from these models in $\S 3$. Finally, in $\oint 4$ we summarize our investigation and discuss the implications on studying $\mathrm{Ly} \alpha$ emission from star-forming galaxies.

\section{MODELS OF ANISOTROPIC NEUTRAL HYDROGEN CLOUDS}

To investigate the effect of anisotropic density and velocity distribution on the $\operatorname{Ly} \alpha$ emission, we construct three simple models of spherical neutral hydrogen gas clouds. The first two models intend to investigate the effects of anisotropy induced by density and velocity separately. The third one is motivated by galactic wind and mimics an outflow confined in a cone. For all the three models, the temperature of the neutral hydrogen atoms in each cloud is fixed at $2 \times 10^{4} \mathrm{~K}$. The Ly $\alpha$ emitting source is assumed to be a point source located at the cloud center.

We emphasize that while our models may capture different aspects of the gas distribution around galaxies, by no means they are realistic. The purpose of the study is to investigate the effects from the anisotropy in the two main quantities that affects $\operatorname{Ly} \alpha$ radiative transfer, i.e., density and velocity. Instead of more sophisticated models with various couplings between density and velocity, we intentionally separate them and build simple models to see the effect from each component.

The first model we consider is a "density gradient" case. In this model, there is no bulk motion of the gas in the cloud and the anisotropic optical depth distribution is purely a result of the anisotropy in the density distribution. Specifically, we introduce a density gradient along the $z$ direction on top of an otherwise uniform cloud. The neutral hydrogen number density $n(z)$ of the cloud is parameterized as

$$
n(z)=\bar{n}\left(1-2 A \frac{z}{R}\right)
$$

where $\bar{n}$ is the mean number density in the cloud, $R$ is the cloud radius, and $A$ is a parameter denoting the magnitude of the density gradient. The column density from the cloud center follows a dipole distribution $N_{\mathrm{HI}}=$ $\bar{n} R(1-A \cos \theta)$, where $\theta$ is the angle with respect to the $+z$ direction (i.e., the polar angle).

The optical depth of Ly $\alpha$ photons depends not only on the density distribution but also on the velocity distribution. Photons of the same frequency appear to have different frequency shifts in the restframe of atoms for atoms with different velocities in the lab frame (i.e., the Doppler effect), which leads to different probability of interacting with the atoms (i.e., different scattering crosssection). Therefore, for the second model, we consider a "velocity gradient" case, where the difference in optical depth along different directions is purely a result of the anisotropy in the velocity distribution. With a uniform density cloud that can undergo uniform Hubble-like expansion/contraction, we introduce a velocity gradient along the $z$ direction,

$$
\mathbf{v}(\mathbf{r})=\frac{r}{R} V \hat{\mathbf{r}}+\frac{z}{R} \Delta V \hat{z}_{+} .
$$

The first term on the right-hand side is the uniform Hubble-like motion, and the second term represents the modification from the velocity gradient, with $\hat{\mathbf{r}}$ and $\hat{z}_{+}$ being the unit vectors along $\mathbf{r}$ and the $+z$ directions, respectively. The quantity $V$ is the Hubble velocity at the edge of the cloud and $\Delta V$ denotes the magnitude of the velocity gradient. A positive (negative) value of $\Delta V$ means that an additional outflow (inflow) field is added on both the $+z$ and $-z$ sides of the cloud.

Finally, motivated by galactic wind, we consider a case named "bipolar wind". For a cloud of uniform density, we set up a Hubble-like velocity field within a limited solid angle,

$$
\mathbf{v}(\mathbf{r})= \begin{cases}\frac{r}{R} V \hat{\mathbf{r}} & \text { if }|z| / r>\mu_{0} \\ 0 & \text { otherwise. }\end{cases}
$$

That is, the "wind" has a half open angle of $\theta_{0}=\cos ^{-1} \mu_{0}$ around the $z$ axis. The model shares some similarity with the "velocity gradient" model. The differences are that here the velocity gradient is imposed along the radial direction (not $z$ direction) and the velocity field is confined to a cone. The geometry is similar to the model in Noterdaeme et al. (2012).

Our models can be regarded as simplistic models of star-forming galaxies emitting Ly $\alpha$ photons with the cloud representing the CGM and IGM environments. With the above models, we consider three cases of mean Ly $\alpha$ optical depth. This can be put in terms of a characteristic column density, $N_{\mathrm{HI}}=\bar{n} R$. The three cases have $N_{\mathrm{HI}}=10^{18}, 10^{19}$, and $10^{20} \mathrm{~cm}^{-2}$, ranging from Ly $\alpha$ limit systems to damped Ly $\alpha$ systems.

For each setup, we perform the radiative transfer calculation of Ly $\alpha$ photons using a Monte Carlo code (Zheng \& Miralda-Escudé 2002). Ly $\alpha$ photons are initially launched isotropically from the cloud center, with frequency following a Gaussian profile centered at the rest-frame Ly $\alpha$ line-center frequency with the width determined by the temperature of the gas. For each run, we use $5 \times 10^{5}-10^{6}$ photons to obtain good statistics on fluxes and spectra. 
Ly $\alpha$ photons are collected once they reach the surface of the cloud. We define the angle $\Theta$ as the polar angle of the escaped photon with respect to $+z$ axis as seen by a distant observer. Since this angle is the most observationally relevant one (and since all three models are axial symmetric about the $z$ axis), we focus our study on the $\operatorname{Ly} \alpha$ emission properties as a function of $\Theta$. For describing the cloud configuration (e.g., density and velocity distribution), we use $\theta$ for the polar angle in the cloud's local frame, i.e., the angle between a given radial direction and the $+z$ axis.

\section{RESULTS OF THE RADIATIVE TRANSFER CALCULATION}

Given the three types of models, different magnitudes of the density/velocity gradient, and different column densities, there are a large number of radiative transfer runs we perform. To obtain a basic picture of the anisotropic Ly $\alpha$ emission, we start with the "density gradient" case with $N_{\mathrm{HI}}=10^{19} \mathrm{~cm}^{-2}$ and introduce our analyses in Ly $\alpha$ flux and spectra. We then present the "velocity gradient" case with $N_{\mathrm{HI}}=10^{19} \mathrm{~cm}^{-2}$ and the "bipolar wind" case. Finally we discuss the general features in the anisotropic $\operatorname{Ly} \alpha$ emission based on all the runs.

\section{1. "Density Gradient" Case with $N_{\mathrm{HI}}=10^{19} \mathrm{~cm}^{-2}$}

We first study the anisotropic Ly $\alpha$ flux seen by distant observers. The flux at a polar angle $\Theta$ is proportional to the number of photons $\Delta N$ in a narrow angular bin $\Delta \Theta$ divided by the corresponding area $2 \pi D^{2} \sin \Theta \Delta \Theta$ for observers at distance $D$. In what follows, we will normalize this flux to the isotropic flux $N /\left(4 \pi D^{2}\right)$. The normalized flux is then

$$
F(\mu)=\frac{2 \Delta N}{N \Delta \mu}
$$

where $\mu=\cos \Theta$. It can also be put as the fractional photon count $\Delta N / N$ divided by the fractional area $\Delta \mu / 2$ around the given polar angle. It satisfies the normalization condition $\int_{-1}^{1} F(\mu) d \mu / 2=1$.

The left panel of Figure 1 shows the angular dependence of the flux and the dependence on the magnitude of the density gradient (with $A=0$ being the uniform sphere case). Resonant scatterings of Ly $\alpha$ photons off neutral hydrogen atoms enable them to probe the optical depths along all directions, and they tend to preferentially make their way out along the path of least resistance. From our setup of the "density gradient" case (Equation 1), the density decreases toward the $+z$ direction. For Ly $\alpha$ photons at the cloud center, the scattering optical depth is lowest (highest) along the $\Theta=0(\Theta=\pi)$ direction. It can be clearly seen from the figure that Ly $\alpha$ photons favor escape along the $\Theta=0$ direction and dislike escape along the $\Theta=\pi$ direction. The ratio of fluxes at $\Theta=0$ and at $\Theta=\pi$ increases as we increase the density gradient, reaching a factor of about 2.5 for $A=0.5$.

To quantify the anisotropy in the full range of the polar angle $\Theta$, we decompose the flux $F(\mu)$ into its multipole components,

$$
F(\mu)=\sum_{l=0}^{\infty} C_{l} P_{l}(\mu)
$$

where $P_{l}$ is the $l$-th order Legendre polynomial. The coefficient $C_{l}$ is solved from the orthogonality of the Legendre polynomials,

$$
C_{l}=\frac{2 l+1}{2} \int_{-1}^{1} F(\mu) P_{l}(\mu) d \mu=\frac{2 l+1}{N} \sum_{i=1}^{N} P_{l}\left(\mu_{i}\right),
$$

where $\mu_{i}$ corresponds to the direction of the $i$-th photon $(i=1,2, \ldots, N)$. The rightmost expression reduces the integral to a simple sum over all photons. It is derived by making use of Equation 4 in the limit of infinitesimally small $\Delta \mu$ bin, and in such a limit, $\Delta N$ is either 1 or 0 . The monopole coefficient $C_{0}$ of $F(\mu)$ is unity by definition.

The density gradient introduces a dipole component in the density distribution (and thus the initial optical depth distribution seen from the center). A corresponding dipole component in the Ly $\alpha$ flux shows up (right panel of Figure 11). However, since Ly $\alpha$ photons encounter a large number of scatterings and continually change their travel directions and frequencies before emerging at the surface, their final distribution does not follow the initial optical depth distribution. As a result, higher multipole components emerge, and their relative contribution increases as the density gradient increases.

Because of the anisotropy, the observed flux depends on the viewing angle (or observed direction). Observers along different directions would infer different luminosities of the source (assuming isotropic emission at the source). Equivalently, for a given observer, observations of similar clouds at random orientations would give the distribution of the observed (apparent) luminosity. In Figure 2, we show the distribution of the ratio of apparent luminosity $L$ to intrinsic luminosity $L_{0}$ as a function of density gradient magnitude. The ratio $L / L_{0}$ is calculated in the same way as $F(\mu)$ in Equation 4. The apparent luminosity is connected to the equivalent width (EW) of Ly $\alpha$ emission. If we neglect other contributions to EW, like dust effect on the continuum and Ly $\alpha$ emission (e.g., Verhamme et al. 2012) and observed Ly $\alpha$ emission being only a fraction of the total Ly $\alpha$ emission (e.g., Zheng et al. 2011b), $L / L_{0}$ is proportional to the Ly $\alpha$ EW. Therefore, the anisotropic emission provides a mechanism for the distribution of Ly $\alpha$ EW.

To be specific and for the convenience of the comparison, here we neglect other contributions and assume that the intrinsic Ly $\alpha \mathrm{EW}$ is $100 \AA$, corresponding to a stellar population with Salpeter initial stellar mass function (IMF) and $1 / 20$ solar metallicity with age above $100 \mathrm{Myr}$ (Malhotra \& Rhoads 2002). The top axis in each panel marks the resultant $\mathrm{EW}=100 \AA\left(L / L_{0}\right)$. Note that the $L / L_{0}(\mathrm{EW})$ distribution is computed based on the Ly $\alpha$ flux distribution shown in the left panel of Figure 1. To reduce the effect of noise in the flux distribution, we use the multipole expansion of the distribution up to to $l=4$ to derive the $L / L_{0}(\mathrm{EW})$ distribution shown in Figure 2.

In the left panel of Figure 2, the original EW distributions from different runs are shown. If the cloud is uniform $(A=0)$, the $\mathrm{EW}$ does not depend on viewing angle and we always have the intrinsic one (i.e., the distribution is just a Dirac $\delta$ function). As the cloud becomes anisotropic, the viewing angle dependent Ly $\alpha$ emission makes the EW distribution extended. The distribution is 

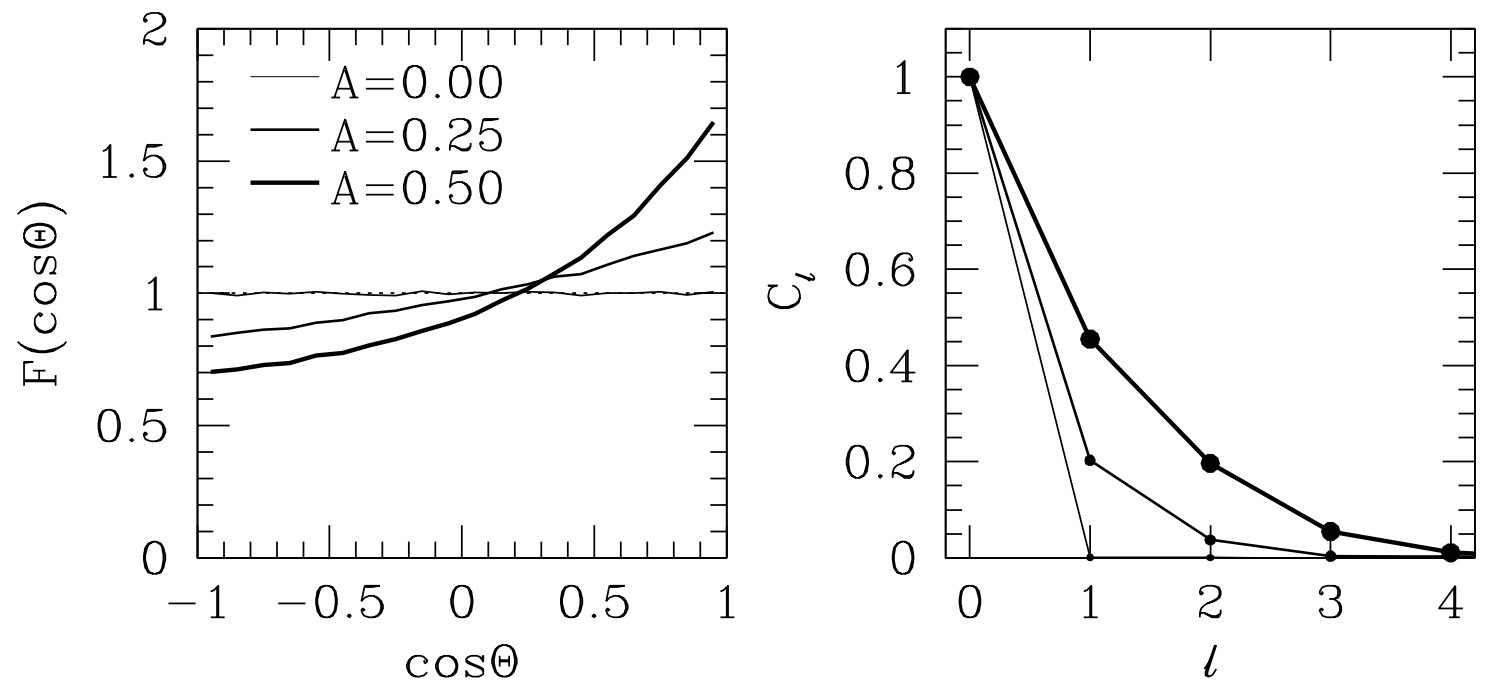

Figure 1. Distribution of $\operatorname{Ly} \alpha$ flux observed by distant observers for a static spherical cloud with anisotropic density distribution (the "density gradient" case with column density of $10^{19} \mathrm{~cm}^{-2}$ ). Left panel: Ly $\alpha$ flux as a function of the polar angle $\Theta$ of the escaping photons. Parameter $A$ denotes the magnitude of the density gradient imposed along the $z$ direction, and the flux is normalized with respect to the isotropic flux of a uniform cloud (i.e., the $A=0$ case). Right panel: the multipole expansion coefficients of the anisotropic distribution of flux. See the text for more details.
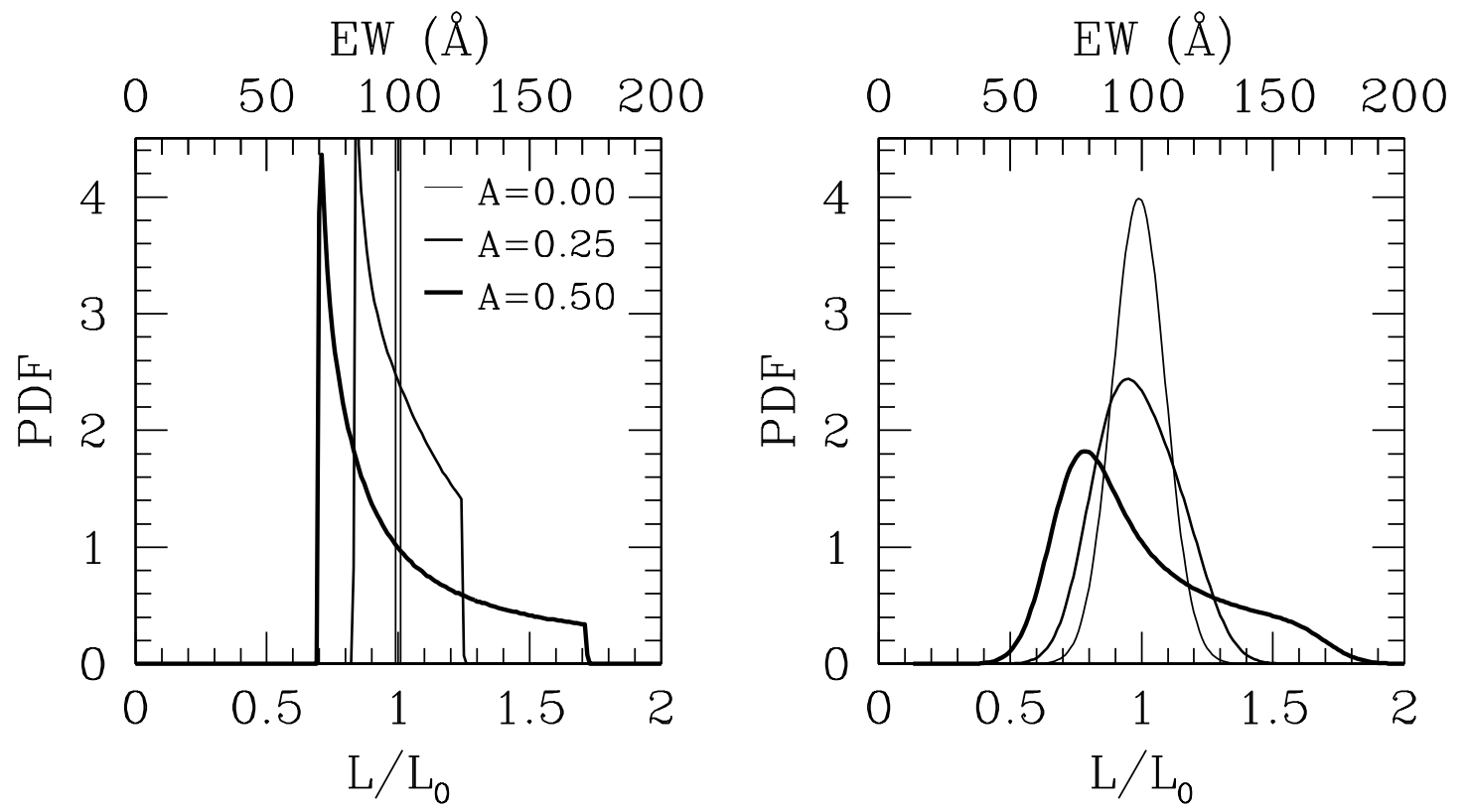

Figure 2. Distribution of apparent (observed) Ly $\alpha$ luminosity from observations along random directions of a static spherical cloud with anisotropic density distribution (the "density gradient" case with column density $10^{19} \mathrm{~cm}^{-2}$ ). The luminosity $L$ is in units of the intrinsic luminosity $\left(L_{0}\right)$. Effectively it can be put in terms of the Ly $\alpha$ EW. In the top axis of each panel, the values of EW are marked by assuming the intrinsic EW to be $100 \AA$ (corresponding to a stellar population with Salpeter IMF and 1/20 solar metallicity with age above $100 \mathrm{Myr}$; see the text). The left panel shows the original distributions, while the right panel shows the ones smoothed with a Gaussian kernel with standard deviation of $10 \AA$ to mimic the effect of measurement errors.

skewed, with a higher amplitude at lower EW. The skewness increases as the anisotropy becomes stronger (as a result of the larger density gradient), and at $A=0.5$ the distribution shows a prominent tail towards high EW values. Interestingly, such a shape of the EW distribution is similar to the observed ones for LAEs (e.g., Ouchi et al. 2008; Nilsson et al. 2009; Ciardullo et al. 2012). The model EW distribution has sharp edges (corresponding to the boundary of $\Theta=0$ and $\pi$ ), which are not seen in real observations. To make a better comparison, we need to take into account the uncertainties in measuring the Ly $\alpha \mathrm{EW}$ in observation. In the right panel of Figure 2, each distribution is smoothed with a Gaussian kernel with standard deviation of $10 \AA$, roughly the size of the measurement errors in Ly $\alpha \mathrm{EW}$ from observation (e.g., Ouchi et al. 2008). The skewness seen in the left panel remains after smoothing, and for strong anisotropic cases, the EW distribution mimics those seen in obser- 

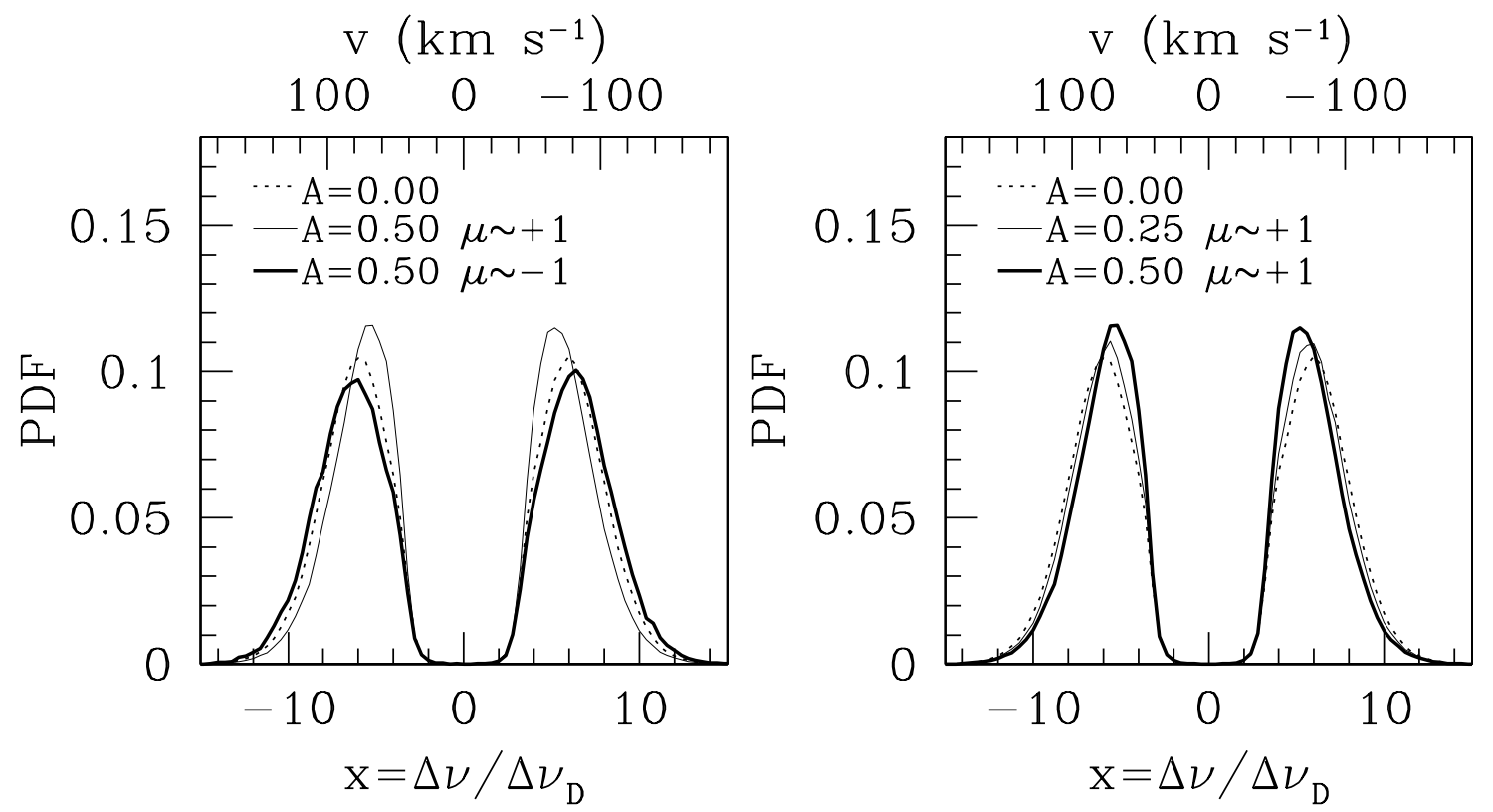

Figure 3. Normalized Ly $\alpha$ spectra from a static spherical cloud with anisotropic density distribution (the "density gradient" case with column density $\left.10^{19} \mathrm{~cm}^{-2}\right)$. Left panel: comparison of spectra observed along the two pole directions $(\mu=\cos \Theta= \pm 1)$ for the $A=0.50$ model. The directions with the lowest and highest column density are $\mu=+1$ and $\mu=-1$, respectively (see Equation 1). Right panel: comparison of spectra observed along one pole direction for clouds with different anisotropic parameter $A$. In both panels, the spectra from the uniform case $(A=0)$ are shown for reference. The frequency offset in units of the Doppler parameter $\Delta \nu_{D}$ and in velocity units are shown in the bottom and top axes, respectively.

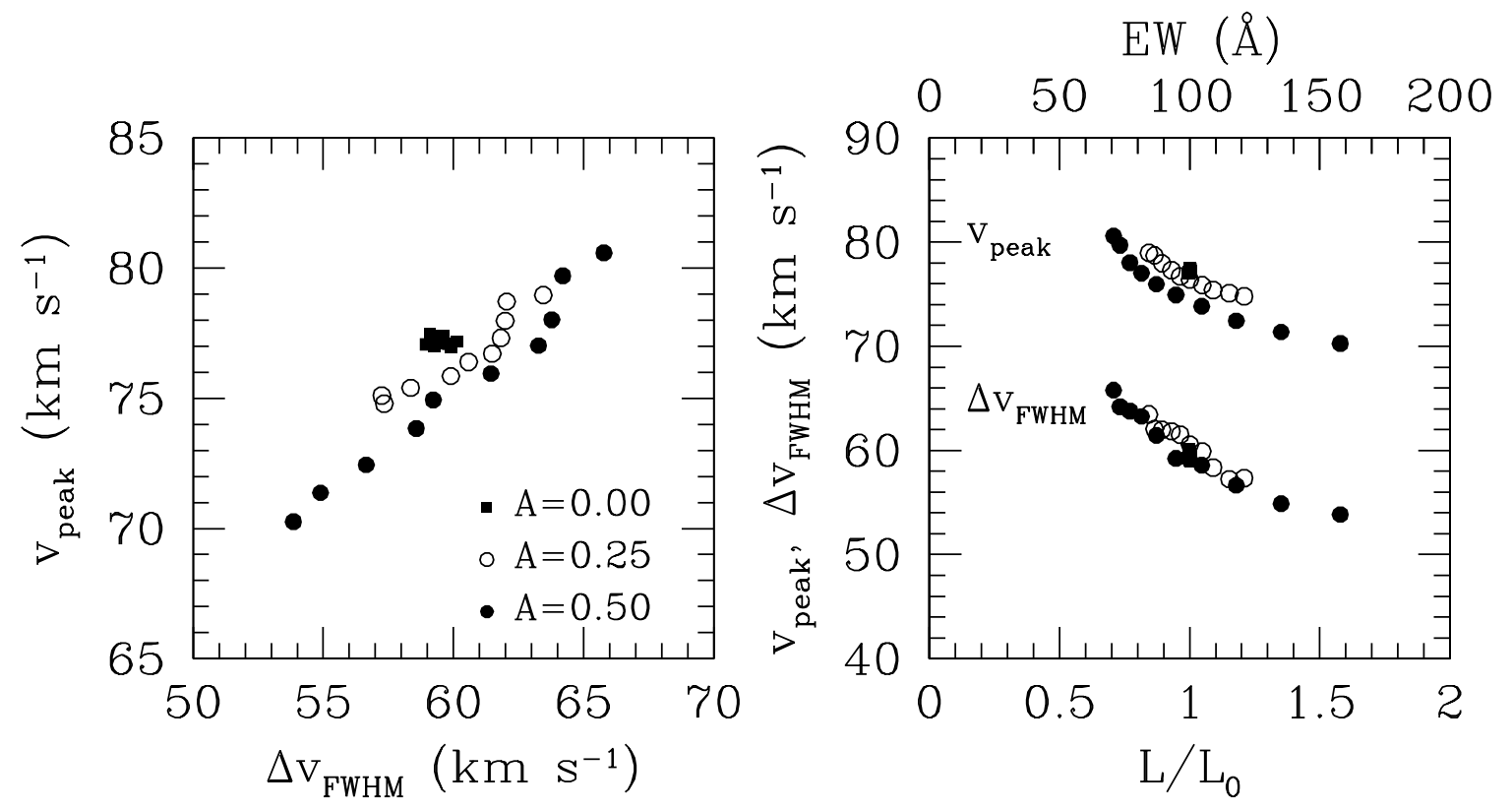

Figure 4. Peak offset $v_{\text {peak }}$ and FWHM $\Delta v_{\text {FWHM }}$ of Ly $\alpha$ emission from a static spherical cloud with anisotropic density distribution (for the "density gradient" case with column density $10^{19} \mathrm{~cm}^{-2}$ ). Left panel: the correlation between $v_{\text {peak }}$ and $\Delta v_{\mathrm{FWHM}}$. Right panel: their correlation with the apparent $\operatorname{Ly} \alpha$ luminosity or Ly $\alpha$ EW. Only the red peak is analyzed here. 
vation.

Besides the viewing angle dependent flux or apparent luminosity, the Ly $\alpha$ spectra are also affected by the system anisotropy. In the left panel of Figure 3, the normalized spectra observed from observers located on the $+z(\Theta=0$ or $\mu=1)$ and $-z(\Theta=\pi$ or $\mu=-1)$ directions for the $A=0.50$ case are compared. The spectra for the isotropic case $(A=0)$ is shown for reference. The bottom axis shows the frequency offset in units the Doppler frequency $\Delta \nu_{D} \equiv\left(v_{p} / c\right) \nu_{0}$, which corresponds to the frequency offset of a line-center photon (with frequency $\left.\nu_{0}\right)$ seen by an atom moving with the most probable thermal velocity $v_{p}=\sqrt{2 k T / m_{H}}$. The top axis marks the offset in velocity units. To escape a static medium, Ly $\alpha$ photons need to shift either to the blue or red wing, where the optical depth is small. This results in a characteristic double-peak profile (e.g., Neufeld 1990; Zheng \& Miralda-Escudé 2002), which applies to the spectra plotted here.

The two opposite directions in the left panel of Figure 3 correspond to the directions of minimum and maximum column density. In general, photons that escape from a direction with a lower column density diffuse less in frequency space. As a consequence, the separation of the two peaks is smaller and the width of each peak is narrower. In the right panel, the spectra in the $\Theta=0$ direction are compared for the $A=0.25$ and $A=0.50$ cases. The $A=0.50$ case has the lower line-of-sight column density, which has narrower peaks with a closer separation. The spectra do not differ substantially from the uniform case (dotted curve). The reason is that the column density modulation on top of the uniform case is only a factor of $1-A \cos \theta$. Even for the $A=0.5$ case, the modulation is only a factor of three. Because of the resonant scattering, Ly $\alpha$ photons can be thought to probe the optical depths in all the directions before escaping along the final direction. This also reduces the difference in the effective column densities experienced by photons escaping along different directions, leading to only small differences in the spectra.

The left panel of Figure 4 shows the viewing angle dependence of two spectral features, the peak offset $v_{\text {peak }}$ with respect to the restframe $\operatorname{Ly} \alpha$ line center and the line width characterized by the full width at half maximum $(\mathrm{FWHM}) \Delta v_{\mathrm{FWHM}}$. In order to reduce the effect of noise, a Gaussian fit around the peak in each spectrum is made to determine the peak offset. Both $v_{\text {peak }}$ and $\Delta v_{\mathrm{FWHM}}$ are computed in ten bins of the viewing angle, with the bottom-left and upper-right points corresponding to $\cos \Theta \sim+1$ and -1 , respectively. We only show the case from the peak in the red side of the spectra - in reality, the blue peak likely becomes insignificant because of the scattering in the IGM with Hubble flow (e.g., Dijkstra. Lidz, \& Wyithe 2007; Zheng et al. 2010; Laursen et al. 2011). The $A=0$ case is shown to give a sense of the noise in our calculation of these quantities from the spectra. There is a clear correlation between $v_{\text {peak }}$ and $\Delta v_{\text {FWHM }}$ - as Ly $\alpha$ photons diffuse more in the frequency space, the spectrum as a whole shifts further away from the initial line center and at the same time it becomes more broadened.

Since both the apparent Ly $\alpha$ luminosity and spectral features depend on viewing angle, there must exist cor- relations between them. In the right panel of Figure 4 , we see that both the peak offset and width are anticorrelated with the Ly $\alpha$ luminosity (or EW). This makes perfect sense - along a direction of easier escape, Ly $\alpha$ emission has a higher flux (thus larger $L / L_{0}$ or $\mathrm{EW}$ ), while Ly $\alpha$ photons diffuse less in frequency space (thus smaller peak offset and width).

As a whole, the resonant scatterings experienced by Ly $\alpha$ photons constantly change their propagation directions. This enables the photons to probe optical depths along all directions, and they tend to escape from the directions with low optical depth. For the "density gradient" case, the anisotropic distribution in the column density of the cloud translates to anisotropic Ly $\alpha$ emission, i.e., viewing angle dependent Ly $\alpha$ emission properties, such as the apparent Ly $\alpha$ luminosity, spectral peak offset, and peak width. The viewing angle dependent Ly $\alpha$ flux leads to a spread in Ly $\alpha$ EW, suggesting an interesting mechanism to produce the observed EW distribution. The emission properties are correlated as a result of the viewing angle dependence. In directions of lower resistance, Ly $\alpha$ photons tend to have higher flux and less diffusion in frequency space.

\section{2. "Velocity Gradient" Case with $N_{\mathrm{HI}}=10^{19} \mathrm{~cm}^{-2}$}

Following the detailed discussions of the "density gradient" case, we now turn to another anisotropic case by adding a velocity gradient to an otherwise static uniform sphere $(V=0$ in Equation 2$)$. The velocity gradient leads to anisotropy in the initial Ly $\alpha$ optical depth, and we expect the Ly $\alpha$ emission to depend on viewing angle as well. As an example, we present the results for the $N_{\mathrm{HI}}=10^{19} \mathrm{~cm}^{-2}$ case.

Figure 5 shows the viewing angle dependence of the flux measured by distant observers (left) and the multipole decomposition (right). The way we add the velocity gradient can be thought as Hubble-like expansion in the $\pm z$ directions and no expansion in the $\pm x$ and $\pm y$ directions. In the plot, $\Delta V$ (taking the values of 50,100 , and $200 \mathrm{~km} \mathrm{~s}^{-1}$ ) is the radial velocity at the edge of the cloud along $\pm z$ directions. For such expansion velocities, the initial Ly $\alpha$ photons launched at the center are effectively at a single frequency (the restframe line-center frequency). In the restframe of the atoms in the expanding cloud, these initial photons appear to be redshifted off the line center, with a frequency shift proportional to the radial velocity. With our setup, the optical depth seen by the initial Ly $\alpha$ photons appears low towards the $\Theta=0$ and $\pi$ directions (poles) and high towards the $\Theta=\pi / 2$ directions (equator). Therefore, unlike the "density gradient" case, here we have a quadrupole-like distribution of the initial optical depth.

Given such a distribution of optical depth, it is expected that photons prefer to escape towards the poles rather than the equator, as seen in the left panel of Figure 5. The ratio of the maximum to minimum fluxes increases from about two for $\Delta V=100 \mathrm{~km} \mathrm{~s}^{-1}$ to about four for $\Delta V=200 \mathrm{~km} \mathrm{~s}^{-1}$. A strong quadrupole component in the angular distribution of flux emerges (right panel of Figure 5), which increases as $\Delta V$ increases. Unlike the "density gradient" case, there is no dipole component from the symmetry of the system. Nevertheless, the density and velocity gradient cases share the same effect on Ly $\alpha$ emission - anisotropy in the initial Ly $\alpha$ 

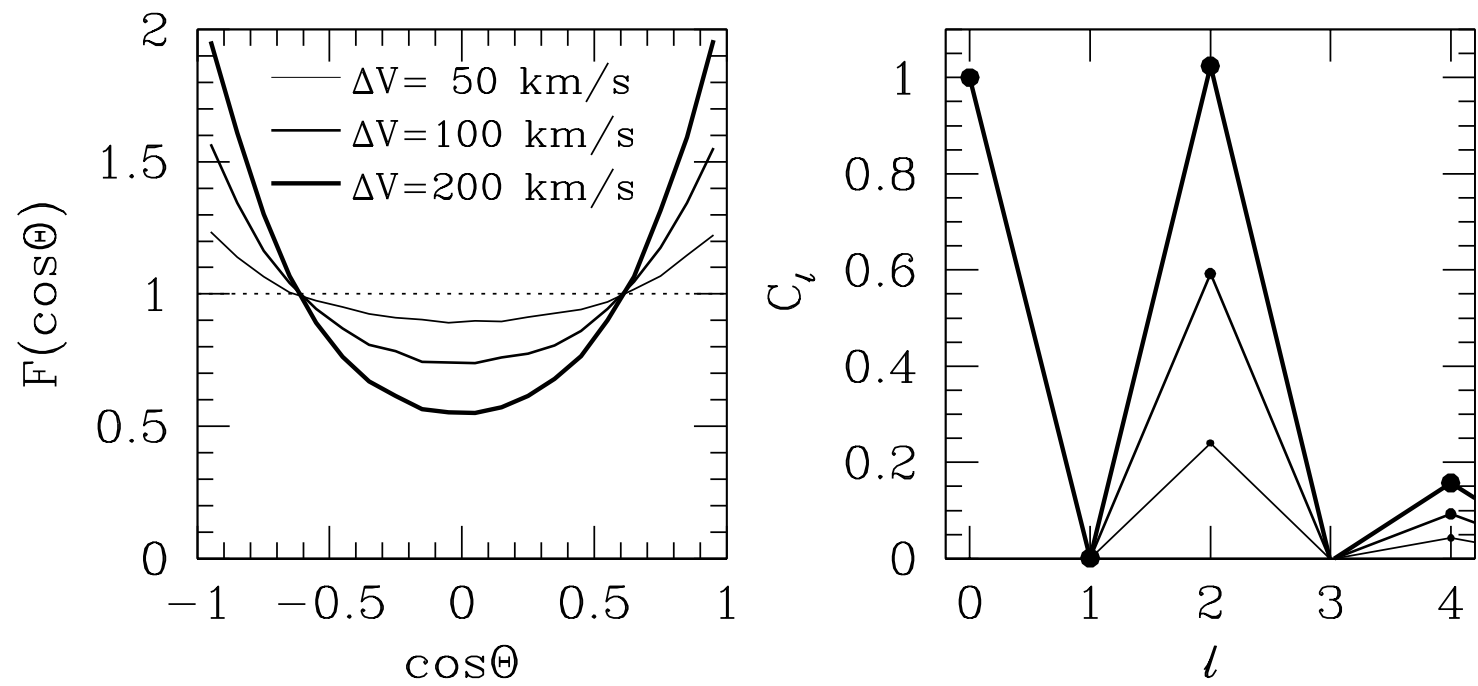

Figure 5. Similar to Figure 1 but for a uniform density cloud with velocity anisotropy (the "velocity gradient" case with column density $10^{19} \mathrm{~cm}^{-2}$ ). The polar angle $\Theta$ is the angle between direction of the distant observer and the $z$ axis. The parameter $\Delta V$, the expansion velocity at the cloud poles, denotes the magnitude of the velocity anisotropy. See text for more details.
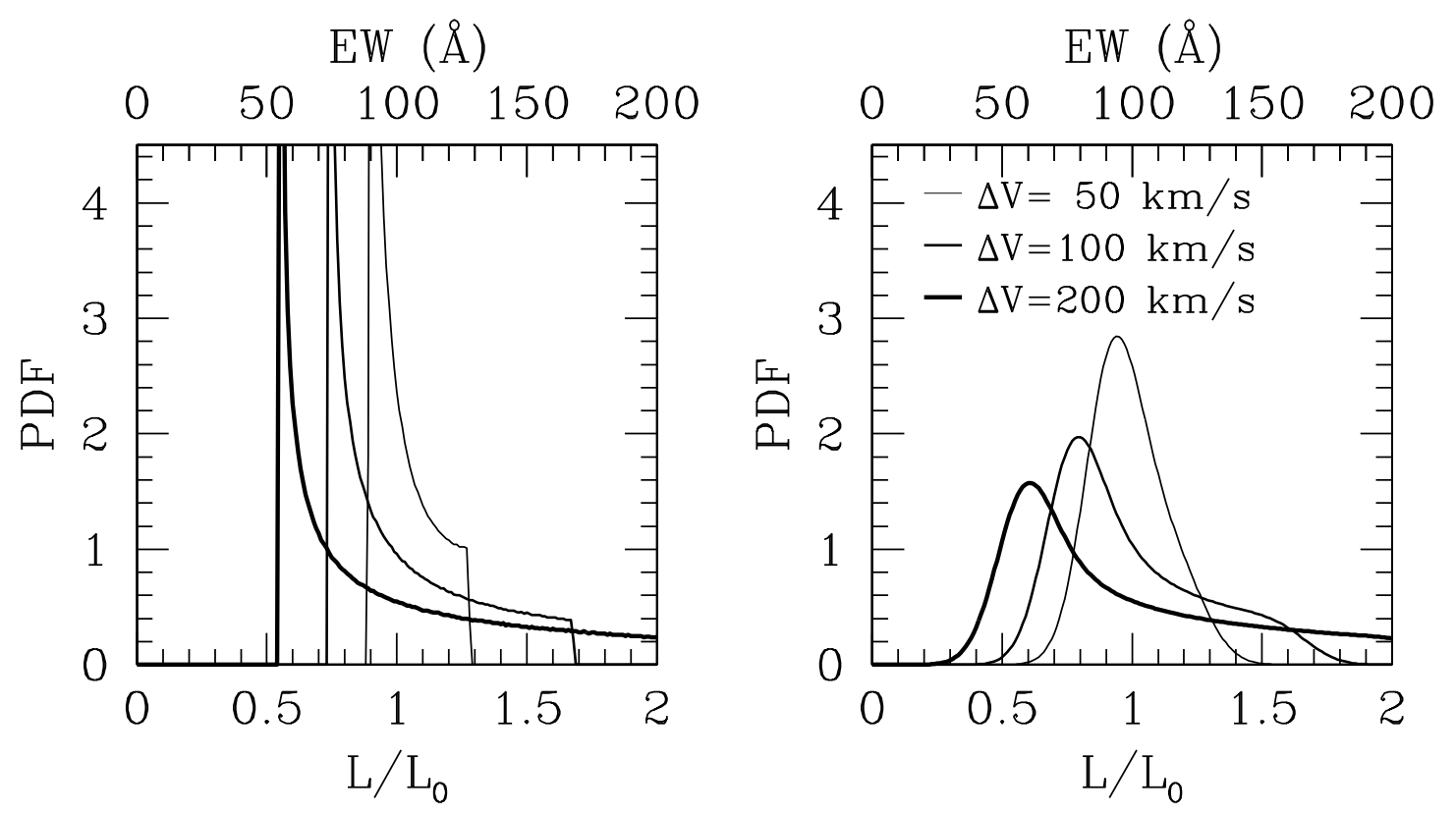

Figure 6. Similar to Figure 2 but for a uniform density cloud with velocity anisotropy (the "velocity gradient" case with column density $10^{19} \mathrm{~cm}^{-2}$ ). The parameter $\Delta V$, the expansion velocity at the poles, denotes the magnitude of the velocity anisotropy. 
optical depth leads to anisotropy in the Ly $\alpha$ flux.

Similar to the "density gradient" case, the anisotropic Ly $\alpha$ emission leads to a skewed distribution of apparent Ly $\alpha$ luminosity $\left(L / L_{0}\right.$ or EW) with a tail of high values (see Figure 6. left for the original distributions and right for the ones smoothed with a Gaussian kernel with a standard deviation of $10 \AA$ ). For $\Delta V=100 \mathrm{~km} \mathrm{~s}^{-1}$ and $200 \mathrm{~km} \mathrm{~s}^{-1}$, the shape of the smoothed distribution looks similar to the observed ones (e.g., Ouchi et al. 2008; Nilsson et al. 2009; Ciardullo et al. 2012).

Figure 7 shows the spectra of photons escaping from the directions of the pole and equator as a function of the velocity gradient (parameterized by the velocity $\Delta V$ at the edge of the cloud). While the spectra in the "velocity gradient" case still have two peaks, they are no longer symmetric about the initial line center (Figure 7). Blue Ly $\alpha$ photons would be redshifted close to the line center in the restframe of hydrogen atoms in an expanding cloud and be strongly scattered. That is, Ly $\alpha$ photons tend to shift redward for an easy escape from the cloud. Overall, the blue peak is suppressed compared to the red peak. The generic features of the spectra can be understood following the interpretations in e.g., Loeb \& Rvbicki (1999), Zheng \& Miralda-Escudé (2002), Dijkstra et al. (2006), and Verhamme et al. (2006), by accounting for the anistropy.

Since both the density and velocity affect the frequency diffusion in the "velocity gradient" case, the relatively tight correlation between the peak position and peak FWHM seen in the "density gradient" case becomes weaker (left panel of Figure 8). So does the correlation between apparent luminosity $L / L_{0}$ (or EW) and FWHM (lower points in the right panel). However, the tight anti-correlation between $L / L_{0}(\mathrm{EW})$ and peak position

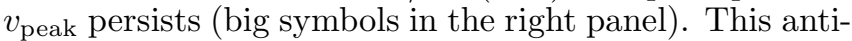
correlation appears to be much stronger than that in the "density gradient" case (Figure 4).

To summarize, for "velocity gradient" induced optical depth anisotropy, Ly $\alpha$ emission again displays the corresponding anisotropy in flux and spectral features. The apparent luminosity or EW distribution closely resembles the observed ones. As an extension, we also study systems with "velocity gradient" in one direction imposed on top of an isotropic Hubble-like expansion, and the relation between EW and $v_{\text {peak }}$ is similar to the "velocity gradient" cases shown here. The results for the extended cases will be presented in $\S 3.4$.

\section{3. "Bipolar Wind" Case}

Before discussing general properties of anisotropic Ly $\alpha$ emission, we briefly present the results on the flux anisotropy for the "Bipolar Wind" case. In this case, the uniform cloud has a Hubble-like expansion within a cone defined by $\theta<60^{\circ}$ and $\theta>120^{\circ}(|\mu|>0.5)$. and remains static outside of the cone. That is, half of the cloud gas is expanding. The expansion velocity at the edge of the cloud is set to be $200 \mathrm{~km} \mathrm{~s}^{-1}$. This specific setup is motivated by the bipolar outflow from galaxies. We perform runs for three column densities: $n R=10^{18}$, $10^{19}$, and $10^{20} \mathrm{~cm}^{-2}$.

The Ly $\alpha$ optical depth is determined by both the column density and velocity. For initial photons with fre- quency $\nu_{i}$, the radial optical depth is

$$
\begin{aligned}
\tau_{\text {ini }} & =\int_{0}^{R} n \sigma\left[\nu_{i}\left(1-\frac{\Delta V}{c} \frac{r}{R}\right)\right] d r \\
& =n R \int_{0}^{1} \sigma\left[\nu_{i}(1-s \Delta V / c)\right] d s
\end{aligned}
$$

where $s=r / R$ and $\sigma$ is the Ly $\alpha$ scattering cross-section. The equation applies to the regions both with and without outflow $(\Delta V \neq 0$ and $\Delta V=0)$. Since all the three runs have the same velocity fields, from the above equation we see that the above three runs have the same fractional anisotropy in the initial Ly $\alpha$ optical depth and they differ in the overall optical depth scale (which is set by the column density).

The "bipolar wind" case shares some similarities with the "velocity gradient" case; the main differences are that its velocity field is radially oriented (rather than only in $\pm z$ direction) and is only within a limited region (rather than the whole cloud). As a consequence, the results on the flux anisotropy, apparent luminosity distribution, and anisotropic spectral properties are also similar to the "velocity gradient" case. For brevity, we only present the flux anisotropy here and have other properties incorporated into the summary of the general results $(\S 3.4)$.

Given the system setup, the anisotropy in the Ly $\alpha$ emission (Figure 9) mainly has a quadrupole component. As the column density increases, Ly $\alpha$ photons experience more scatterings and more changes in travel directions. The anisotropy in the initial Ly $\alpha$ optical depth is therefore becomes less important, and as a consequence the anisotropy in Ly $\alpha$ flux becomes weaker.

\subsection{General Results}

With the three cases discussed in the previous subsections, we turn to present the general results and make an attempt to further connect to observations.

A few more model runs are included here. We extend the "velocity gradient" case by imposing a velocity gradient along the $z$ direction on top of a spherical cloud with Hubble-like expansion (see Equation 2). The Hubble-like expansion has a velocity $V=100 \mathrm{~km} \mathrm{~s}^{-1}$ at the edge of the cloud and we impose different velocity gradients with the parameter $\Delta V$ ranging from -100 to $100 \mathrm{~km} \mathrm{~s}^{-1}$ with a step size $50 \mathrm{~km} \mathrm{~s}^{-1}$. We also perform runs with different column densities $\left(n R=10^{18}, 10^{19}\right.$, and $\left.10^{20} \mathrm{~cm}^{-2}\right)$ for the "density gradient" case and the "velocity gradient" case and its extension. In total, the results of 32 runs from all the cases are presented here.

Overall, we find system anisotropy leads to anisotropies in Ly $\alpha$ emission. The observed Ly $\alpha$ luminosity depends on the viewing angle. Since higher luminosities are likely observed along directions of easier escape, we expect the luminosity to show a correlation with the optical depth of the same direction. However, we do not expect the correlation to be tight. Ly $\alpha$ photons probe the optical depth in all directions in a convoluted way, since their scattering with the neutral hydrogen atoms causes their positions, directions, and frequencies to continually change. Furthermore, for observed/apparent Ly $\alpha$ luminosity, the absolute value of optical depth is not a good indicator. As an example, consider the uniform sphere case. For the same intrinsic luminosity, spheres of different column 

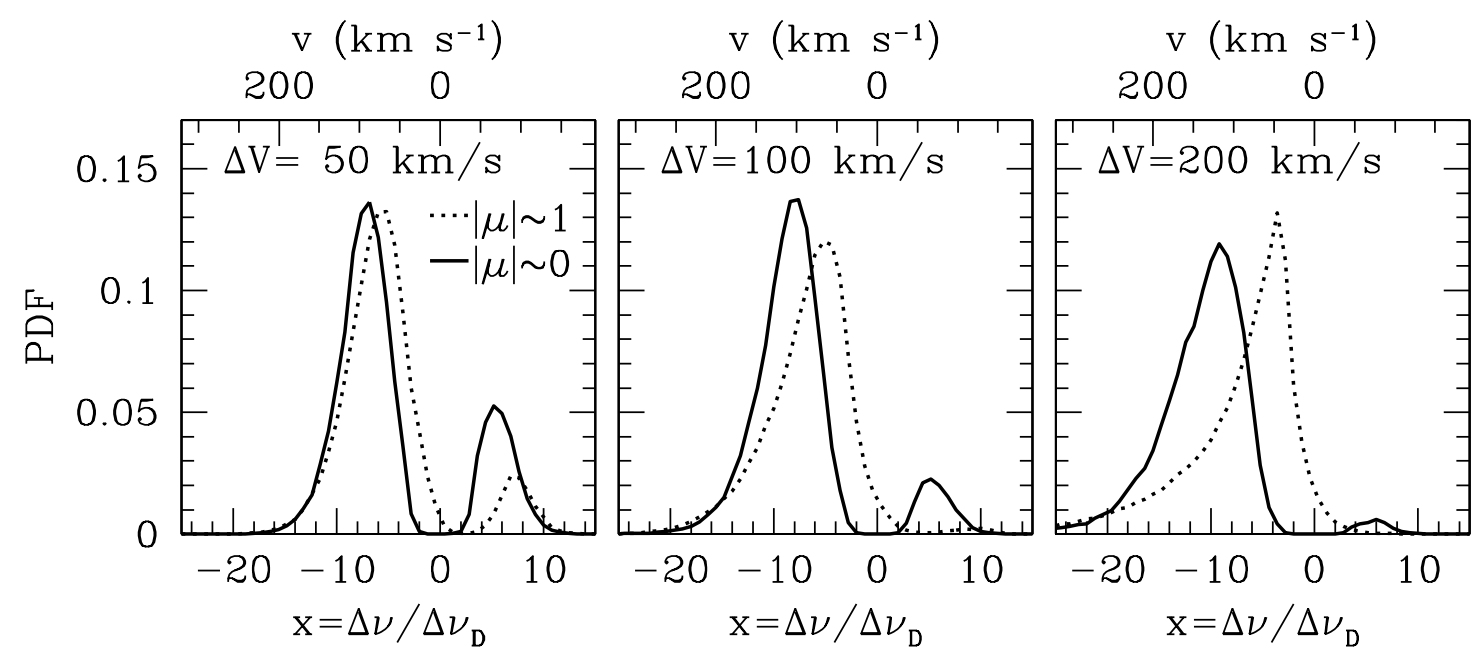

Figure 7. Similar to Figure 3 but for a uniform density cloud with velocity anisotropy (the "velocity gradient" case with column density $10^{19} \mathrm{~cm}^{-2}$ ).

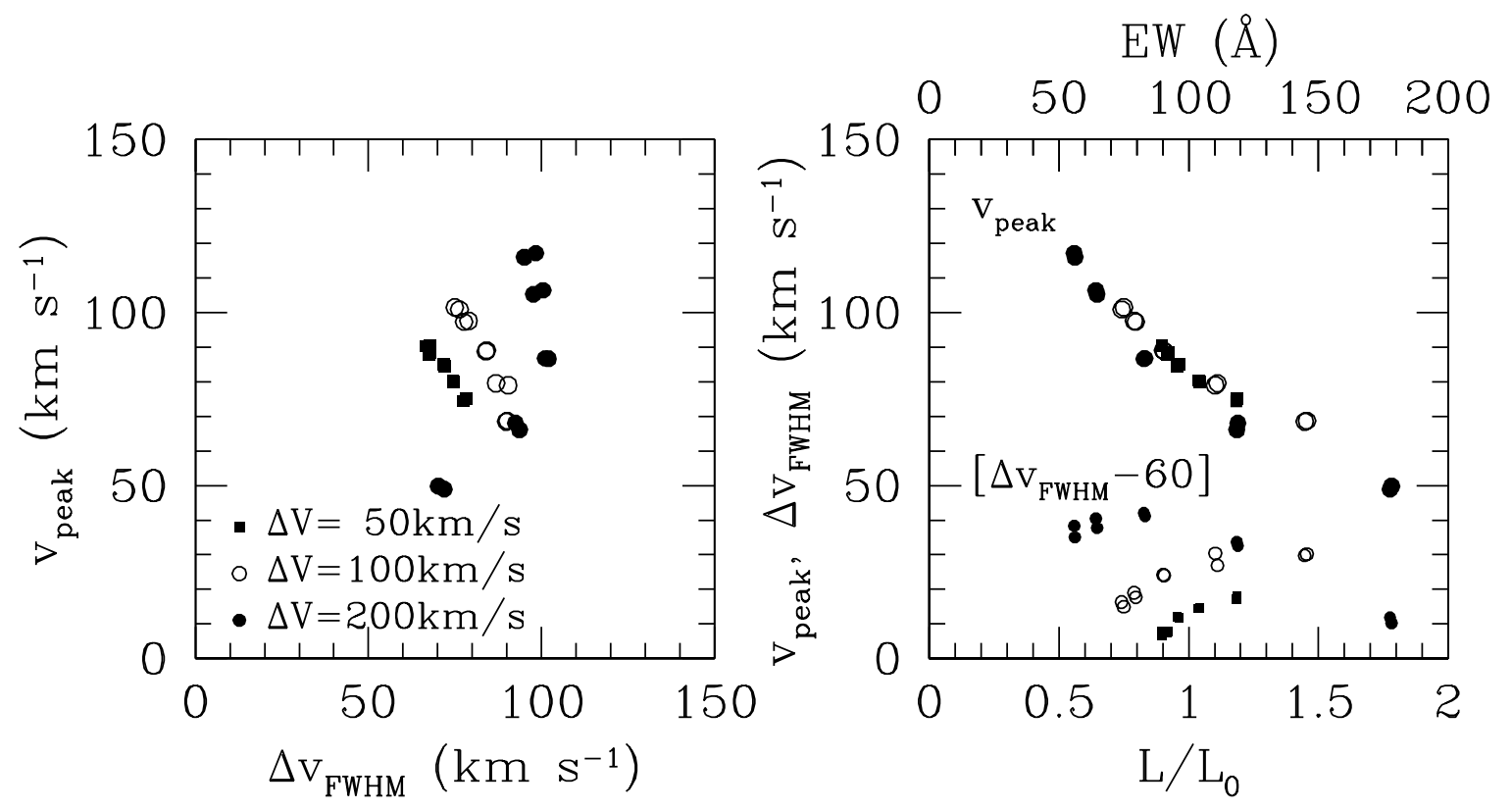

Figure 8. Similar to Figure 4 but for a uniform density cloud with velocity anisotropy (the "velocity gradient" case with column density $10^{19} \mathrm{~cm}^{-2}$ ). 

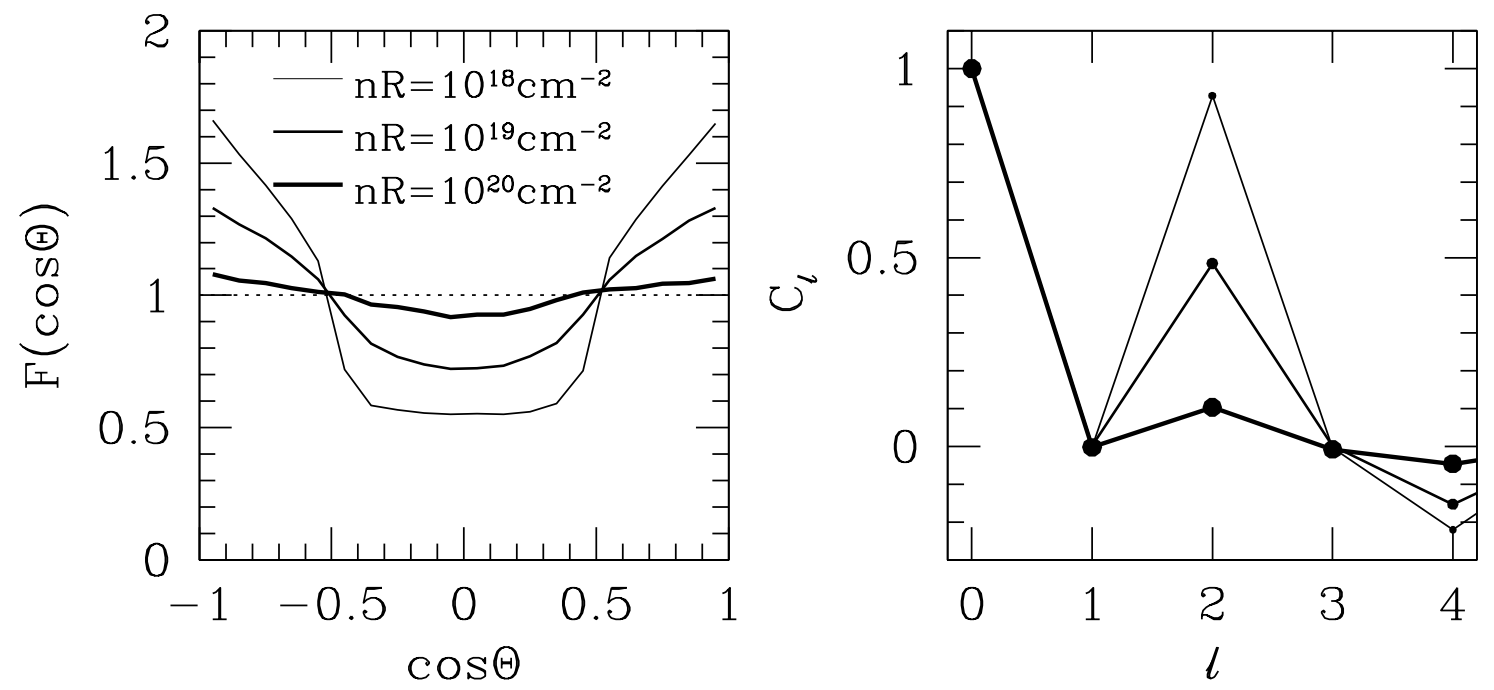

Figure 9. Similar to Figure 5 but for a uniform density cloud with bipolar outflows (the "bipolar wind" case).

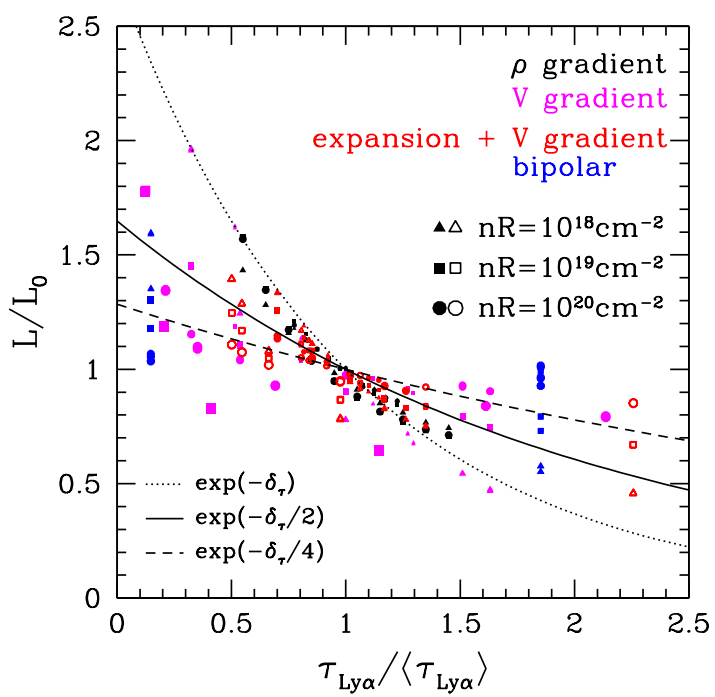

Figure 10. The relation between the apparent Ly $\alpha$ luminosity and the relative initial line-center optical depth. The value $\left\langle\tau_{\text {Ly } \alpha}\right\rangle$ is the line-center optical depth for initial $\operatorname{Ly} \alpha$ photons, averaged over all directions. The curves show $\exp \left(-\delta_{\tau}\right), \exp \left(-\delta_{\tau} / 2\right)$, and $\exp \left(-\delta_{\tau} / 4\right)$ to guide the eye, where $\delta_{\tau}$ is the optical depth excess defined as $\delta_{\tau} \equiv \tau_{\mathrm{Ly} \alpha} /\left\langle\tau_{\mathrm{Ly} \alpha}\right\rangle-1$. The anisotropy models include those caused by density anisotropy ("density gradient" case; black points), by velocity anisotropy ("velocity gradient" case; magenta points) and its extension with an additional isotropic expansion component ("expansion + velocity gradient"; red points), and by bipolar outflow ("bipolar wind" case; blue points). Systems with three different column densities are studied for each case, $10^{18}$ (triangles), $10^{19}$ (squares), and $10^{20} \mathrm{~cm}^{-2}$ (circles). Open and filled symbols denote setups with negative and positive velocity gradient imposed, respectively.

density (optical depth) have the same observed luminosity, since all Ly $\alpha$ photons escape 1 A better quantity that connects to luminosity could be the relative optical depth among all directions.

1 This particular example shows that simply applying a $\exp \left(-\tau_{\nu}\right)$ correction to the initial Ly $\alpha$ spectra is not the right way to do the Ly $\alpha$ radiative transfer, where $\tau_{\nu}$ is the frequency dependent Ly $\alpha$ optical depth along the line-of-sight direction. This is demonstrated and emphasized in Zheng et al. (2010).

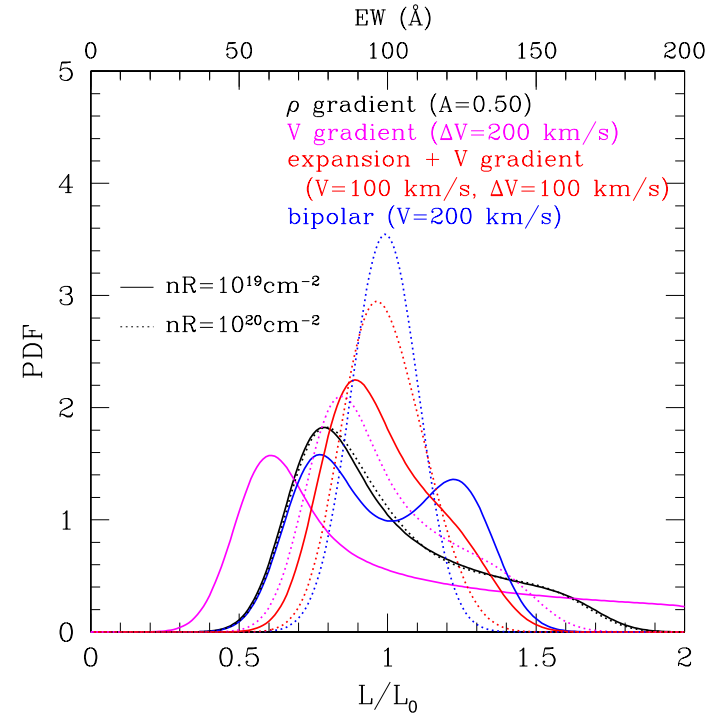

Figure 11. Distribution of apparent (observed) Ly $\alpha$ luminosity (or EW) from a few selected models. The curves have been smoothed with a Gaussian kernel with standard deviation of $10 \AA$ to mimic the effect of measurement errors.

In Figure10, we plot the observed luminosity $L$ in units of the intrinsic luminosity $L_{0}$ as a function of the relative optical depth $\tau_{\text {Ly } \alpha} /\left\langle\tau_{\text {Ly } \alpha}\right\rangle$ for all the models. Here $\tau_{\text {Ly } \alpha}$ is the initial line-center optical depth for Ly $\alpha$ photons and $\left\langle\tau_{\mathrm{Ly} \alpha}\right\rangle$ is the average over all directions. Or equivalently we can define the fractional excess $\delta_{\tau}=\tau_{\text {Ly } \alpha} /\left\langle\tau_{\text {Ly } \alpha}\right\rangle-1$ in the initial line-center optical depth. The three curves are shown in the form of $\exp \left(-\delta_{\tau}\right), \exp \left(-\delta_{\tau} / 2\right)$, and $\exp \left(-\delta_{\tau} / 4\right)$ to guide the eye. Clearly there exists an overall correlation between apparent luminosity (or EW) and the relative line-center optical depth. The plot serves as a nice summary of the anisotropic $\operatorname{Ly} \alpha$ emission from all the models considered in this paper. It depicts the idea that Ly $\alpha$ photons prefer to escape along the direction of least resistance. The resonant scatterings allow Ly $\alpha$ photons to probe optical depth along different directions, and the key quantity that determines the final anisotropic distribution of $\operatorname{Ly} \alpha$ emission is the relative 
optical depth, not the absolute one.

As a result of the anisotropy, the observed Ly $\alpha$ luminosity from a set of randomly oriented clouds will spread out around the intrinsic luminosity. Figure 11$]$ summarizes the distributions of the apparent $\mathrm{Ly} \alpha$ luminosity (EW) by showing the result from a few selected models. The apparent luminosity/EW distribution is largely determined by the anisotropy (in the initial optical depth) of the system. For a fixed anisotropy factor (e.g., density or velocity gradient), a cloud with higher column density has a lower degree of anisotropy in the initial optical depth distribution. This translates to smaller spread in the apparent luminosity/EW distribution (e.g., comparing the $10^{19} \mathrm{~cm}^{-2}$ cases with those of $\left.10^{20} \mathrm{~cm}^{-2}\right)$. The anisotropy leads to a skewed EW distribution with a tail toward higher values. It becomes more skewed with higher degrees of flux anisotropy (i.e., lower column density). For the high column density "bipolar wind" case, a low amplitude bump is seen at higher EW values. For this model, the superposition of the EW distributions from systems with different column densities (more abundant for lower column density) can produce a distribution with an extended tail. The skewed EW distribution looks similar to the observed ones (e.g., Ouchi et al. 2008; Nilsson et al. 2009; Ciardullo et al. 2012), which implies that anisotrpic Ly $\alpha$ emission provides one mechanism to contribute to the Ly $\alpha$ EW distribution.

The left panel of Figure 12 summarizes the relation between Ly $\alpha$ EW (or the apparent luminosity $L / L_{0}$ ) and the shift in the (red) peak of the Ly $\alpha$ line. The points are clearly grouped according to the column density (triangles, squares, and circles for $n R=10^{18}, 10^{19}$, and $10^{20} \mathrm{~cm}^{-2}$, respectively), with larger peak shift at higher column density. For the sequence at a fixed column density, the spread in the EW distribution results from the dependence on the viewing angle. The size of the symbols indicates the magnitude of the anisotropy of the system, with larger symbols for higher anisotropy.

For the "density gradient" case (black points), the anticorrelation between EW and $v_{\text {peak }}$ is stronger at higher column density, but overall the anti-correlation is weak. The offset in the peak position is largely determined by the column density. At fixed column density, a cloud with a low anisotropy in the initial optical depth (indicated by smaller symbols) has a smaller spread in the apparent luminosity/EW distribution viewed from different directions, which is expected.

For the "velocity gradient" case (magenta points), a similar trend of increasing strength of EW- $v_{\text {peak }}$ anticorrelation with increasing column density is found, but the anti-correlation is much stronger than the "density gradient" case. At fixed velocity gradient, a higher column density means a lower degree of system anisotropy, and therefore we see a smaller spread. At fixed column density, a smaller velocity gradient (indicated by smaller symbols) also means a lower degree of system anisotropy, leading to smaller spread in EW.

The extended "velocity gradient" case (red points) closely follows the above results, with filled (open) symbols for imposing positive (negative) velocity gradients. Similar results are also found for the "bipolar wind" case (blue symbols).

As a whole, for the runs we have performed, the $\operatorname{Ly} \alpha$ line peak offset is mainly driven by the column density, with larger offset from systems of higher column density. The overall trend seen in the left panel of Figure 12 from all the cases we consider is that the spread in EW distribution decreases with increasing Ly $\alpha$ line peak offset.

Interestingly the above trend appears to be consistent with recent observations. In the right panel of Figure 12 , we reproduce the data points compiled and analyzed by Hashimoto et al. (2013) for LAEs, Ly $\alpha$ blobs (LABs), and Lyman break galaxies (LBGs). The EW can be measured from the Ly $\alpha$ line luminosity and continuum with narrow-band and broad-band photometry. To determine the peak offset, the systemic redshifts of the galaxies are measured from nebular emission lines such $\mathrm{H} \alpha$. Even though Hashimoto et al. (2013) cast their result as an anti-correlation between $\mathrm{EW}$ and $v_{\text {peak }}$, the plot suggests a smaller EW spread towards larger peak offset. We emphasize that we do not intend to fit the observation here, since our models are simplistic. Nevertheless, it is encouraging to see that both the ranges of EW and $v_{\text {peak }}$ and their relation fall into the ballpark of the observational results. It suggests that the key element in our simple models (i.e., Ly $\alpha$ emission from anisotropic systems) could play an important role in shaping the Ly $\alpha$ emission in real systems. More realistic models (e.g., those based on galaxy formation simulations) are necessary for a better understanding and comparison with the observation.

To study the $\mathrm{EW}-v_{\text {peak }}$ relation observationally, the quantity $v_{\text {peak }}$ is the more difficult one to measure. The reason is that a line indicating the systemic redshift of the galaxy is needed. Examples of such lines are $\mathrm{H} \alpha$ and [O III] (e.g., Steidel et al. 2010; McLinden et al. 2011; Hashimoto et al. 2013), which shift to infrared for high redshift galaxies. It would be interesting to see whether there are other possible ways that can inform us about $v_{\text {peak }}$, without the knowledge of the systemic redshift. Based on our results, we see a general correlation between $v_{\text {peak }}$ and the FWHM $\Delta v_{\text {FWHM }}$, mainly driven by the column density. The degree of correlation varies from case to case, which leads to a large scatter among cases with different anisotropy setups. We investigate whether other spectral features of the Ly $\alpha$ line can help to reduce the scatter. Besides line peak offset and the FWHM of the line, another obvious property of the Ly $\alpha$ line is its asymmetry. Both from observation and from our model, the red peak of the Ly $\alpha$ line is usually asymmetric, having a red tail. We therefore can introduce an asymmetry parameter. It can be defined by comparing the line fluxes or widths blueward and redward of the line peak (see, e.g., Rhoads et al. 2003). Here we define it as three times the ratio of the widths at half maximum at the blue and red sides of the peak, $f_{\text {asym }}=3 W_{\text {blue }} / W_{\text {red }}$. By introducing this parameter, we find a relatively tight correlation between $v_{\text {peak }}$ and $\Delta v_{\text {FWHM }} f_{\text {asym }}$ (see Figure 13), probably with the low $v_{\text {peak }}$ part of the "bipolar wind" model causing the largest scatter. Note that both $\Delta v_{\text {FWHM }}$ and $f_{\text {asym }}$ can be inferred from the Ly $\alpha$ spectra. Observationally, McLinden et al. (2013) show a possible trend of increasing line asymmetry with increasing peak offset. It would be interesting to see whether the correlation seen in our study holds for realistic models and whether observations support it. Note that for a fair comparison with observations, the spectral reso- 

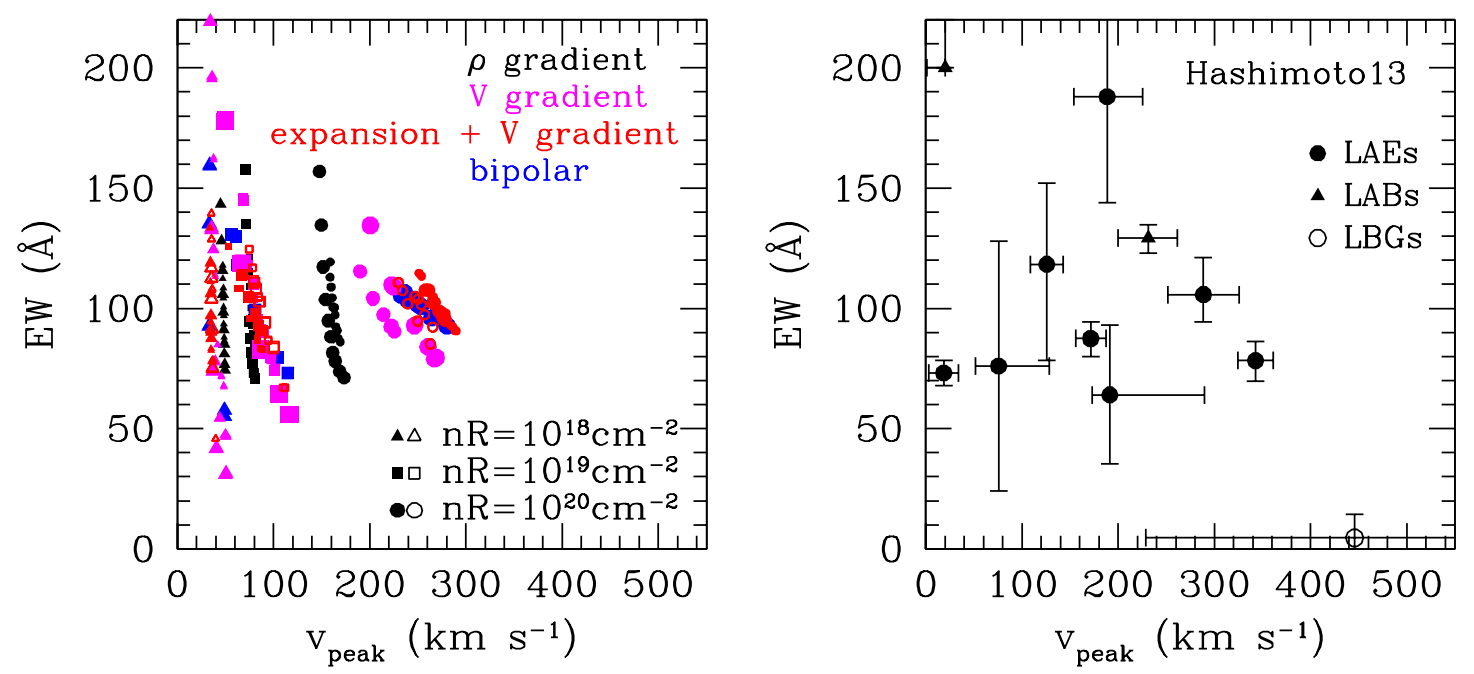

Figure 12. The relation between the Ly $\alpha$ EW and Ly $\alpha$ line peak offset $v_{\text {peak }}$. Left panel: the relation from our models of anisotropic clouds, including those caused by density anisotropy ("density gradient" case; black points), by velocity anisotropy ("velocity gradient" case; magenta points) and its extension with an additional isotropic expansion component ("expansion + velocity gradient"; red points), and by bipolar outflow ("bipolar wind" case; blue points). Systems with three different column densities are studied for each case, $10^{18}$ (triangles), $10^{19}$ (squares), and $10^{20} \mathrm{~cm}^{-2}$ (circles). At a given column density of each case, the symbol size indicates the degree of anisotropy of the system (larger for stronger anisotropy). Open and filled symbols denote cases with negative and positive velocity gradient imposed, respectively. Right panel: the observed relation. The data are taken from those compiled and analyzed in Hashimoto et al. (2013).

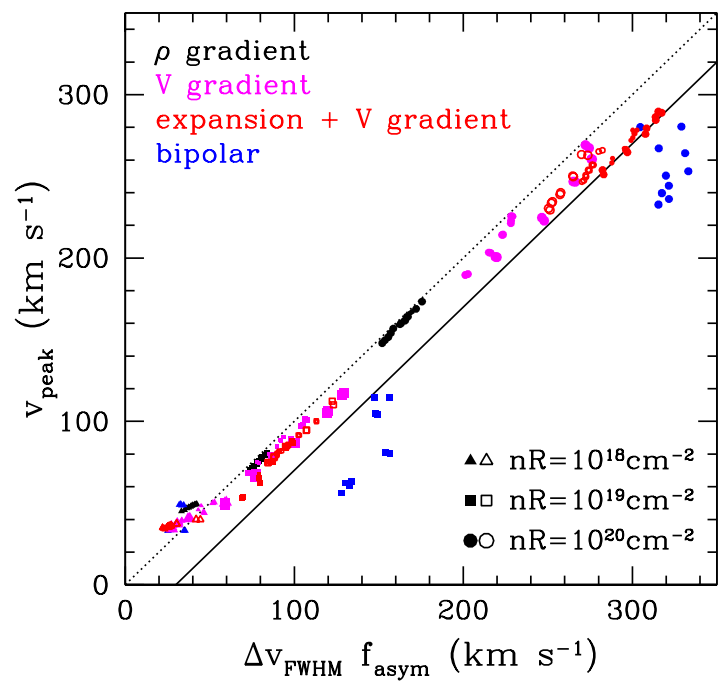

Figure 13. The relation between the Ly $\alpha$ line peak offset $v_{\text {peak }}$ and the FWHM of the line $\Delta v_{\mathrm{FWHM}}$ modified by the line asymmetry parameter $f_{\text {asym }}$. The asymmetry parameter $f_{\text {asym }}$ is defined as three times the ratio of the width at half maximum on the blue and red sides of the line peak. The dotted line denotes the equality of the two quantities, while the solid line has a $30 \mathrm{~km} \mathrm{~s}^{-1}$ downward offset to approximate the mean relation. The symbols have the same meanings as in Figure 12

lution needs to be taken into account. Its effect is to smooth the Ly $\alpha$ line and increase both the FWHM and the asymmetry parameter, which would cause a slope change for the correlation in Figure 13. In any case, a correlation of similar type to ours or with similar spirit is worth pursuing. It can not only provide a way to determine the peak offset without knowing the systemic redshift, but also serve as a relation to test theoretical models of environments of star-forming galaxies.

\section{SUMMARY AND DISCUSSION}

We perform a theoretical investigation of the effect of anisotropy in neutral hydrogen systems on the observed properties of Ly $\alpha$ emission. The motivation of the work is to help understand the relation between the gas environment around star-forming galaxies and Ly $\alpha$ emission properties and yield insights on using the latter to probe the former. We find that the anisotropy in the spatial and kinematic distributions of neutral hydrogen can play an important role in shaping the observed Ly $\alpha$ emission properties from star-forming galaxies.

We consider simple configurations of neutral hydrogen systems of spherical clouds with a central point source of Ly $\alpha$ emission. We set up system anisotropy induced by different factors. The term "system anisotropy" refers to the anisotropy in the initial optical depth of Ly $\alpha$ photons. Since the scattering optical depth of Ly $\alpha$ photons depends on both density and velocity, we basically explore anisotropies induced by two types of causes. The first type is density-caused and a "density gradient" is applied to the cloud along one direction. The second type is velocity-caused. For the "velocity gradient" case, we apply a velocity gradient along one direction to the otherwise static cloud. Its extension, the "expansion plus velocity gradient" case, has an additional, isotropic Hubble-like expansion. The "bipolar wind" case, which has a spatially separated outflowing region, also belongs to this velocity-caused category. For each case, we set up systems of different column densities and of different degrees of anisotropy. We perform Monte Carlo Ly $\alpha$ radiative transfer calculations to obtain the Ly $\alpha$ emission escaping from the clouds and study the anisotropy in Ly $\alpha$ flux and spectral features.

Owing to the resonant scatterings with neutral hydrogen atoms, a Ly $\alpha$ photon takes a random walk in a cloud, with its traveling direction and frequency constantly changing. Such a random walk enables the photon to explore the optical depths along different direc- 
tions. It tends to escape along the directions of low resistance. An initial anisotropy in Ly $\alpha$ scattering optical depth therefore translates to the anisotropy in the escaping Ly $\alpha$ emission. It is not surprising that a dipole (quadrupole) component in Ly $\alpha$ flux (or apparent Ly $\alpha$ luminosity) is found for a dipole (quadrupole) component in the system optical depth anisotropy. Roughly speaking, the Ly $\alpha$ flux in a direction is determined by the optical depth along that direction relative to those along other directions, or by the fractional excess of the initial optical depth.

For an ensemble of the same systems with random orientations, the anisotropic Ly $\alpha$ emission gives rise to a non-trivial distribution in the observed/apparent Ly $\alpha$ luminosity or the Ly $\alpha$ EW. The general EW distributions from our models are found to be skewed with a tail towards high EW values. Such distributions, especially the ones from the "density gradient" and "velocity gradient" cases, resemble the observed ones for LAEs (e.g., Ouchi et al. 2008; Nilsson et al. 2009; Ciardullo et al. 2012). The observed Ly $\alpha$ EW distribution is likely a superposition of systems of a variety of environments. Given the generic features seen in our models, a superposition of EW distributions from models with different types of anisotropy, different degrees of anisotropy, and different column densities will still keep the skewed shape and resemble the observed distributions. Even though our models are idealized and hardly realistic, our results suggest that the viewing angle dependent $\operatorname{Ly} \alpha$ emission caused by system anisotropy can be an important factor in contributing to the EW distribution of LAEs.

Ly $\alpha$ spectra are also subjected to the anisotropic effect. Noticeably for a given system, the offset of the line peak depends on the viewing angle. Ly $\alpha$ photons escaping from the directions of low optical depths typically have a spectrum with a smaller peak offset from the line center. The overall scale of the offset depends strongly on the mean column density of the cloud. The viewing angle dependence of both the apparent luminosity (EW) and peak offset cause an anti-correlation between the two quantities for a given cloud - along the direction that is easy to escape, we have a higher Ly $\alpha$ flux (apparent luminosity) and Ly $\alpha$ photons diffuse less in frequency space. We note that the peak offset is not necessarily a feature specific to the anisotropic systems we study, and it can also occur in isotropic systems. In such systems, the peak offset increases with increasing system column density or optical depth. However, for isotropic clouds with a given intrinsic Ly $\alpha$ luminosity $L_{0}$, the apparent luminosity $L=L_{0}$, which is independent of the column density. Therefore, the anti-correlation between peak offset and luminosity (EW) we find in the anisotropic systems cannot be naturally produced by the isotropic systems, unless with the effect of dust invoked or with a contrived scenario of putting more luminous sources in systems of lower column densities.

For a fixed magnitude of the anisotropy factor (e.g., density or velocity gradient), the system anisotropy becomes weaker at higher column density. From the viewing angle dependence of Ly $\alpha$ luminosity or EW at fixed column density and the dependence of the peak offset on column density, we find that at larger peak offset the spread in the apparent luminosity or EW is reduced. Interestingly this generic feature between EW and peak offset in the model anisotropic Ly $\alpha$ emission is seen in the observational data in Hashimoto et al. (2013), suggesting that anisotropic Ly $\alpha$ emission can be at work in real Ly $\alpha$ emitting systems.

Based on our simple models, we also find a correlation between the $\operatorname{Ly} \alpha$ line peak offset and line shape (i.e., some combination of the FWHM and asymmetry of the line). An observational test of this correlation could help us understand Ly $\alpha$ radiative transfer in real systems by comparing model predictions and observations. Conversely, a similar kind of relation between Ly $\alpha$ peak offset and spectral shape established from observation, if possible, would provide opportunities for us to learn about the density/velocity structures or, more generally, the environment around Ly $\alpha$ emitting systems that shapes the Ly $\alpha$ emission properties. Additionally, this type of correlation has the potential use of determining the systemic redshift of galaxies with the Ly $\alpha$ emission line alone.

Our investigation based on analytic models suggests that anisotropies in the spatial and kinematic distributions of neutral hydrogen in the CGM and IGM can be an important ingredient in determining the properties of observed Ly $\alpha$ emission. While we try to build models that capture some features in the CGM and IGM around star-forming galaxies (e.g., density inhomogeneity and outflow), they are simplistic and by no means realistic. A further study along this path is to apply similar analyses to galaxies in high resolution cosmological galaxy formation simulations. If the relations found in this paper turn out to exist for simulated galaxies, we expect the scatter to be large given the more complex environment around galaxies.

In fact, some of the features found in our study are broadly similar to those from studies of individual simulated galaxies. For example, the viewing angle dependent Ly $\alpha$ flux (e.g., Laursen et al. 2009; Zheng et al. 2010; Barnes et al. 2011; Yajima et al. 2012; Verhamme et al. 2012), correlation of flux with the initial Ly $\alpha$ optical depth (e.g., Figure 11 in Zheng et al. 2010), and the relation between peak shift and Ly $\alpha$ flux (e.g., Figure 9 in Zheng et al. 2010). Compared to the results from our simple models, the viewing angle dependent Ly $\alpha$ flux in the above study does show much larger spread, an expected consequence of the more complex density and velocity distribution of the gas around galaxies.

One can certainly extend our simple models to more complicated models, with more realistic geometry and coupling between density and velocity, which is beyond the scope of this paper. Indeed, models with various setups start to be studied (e.g., Laursen et al. 2013; Behrens et al. 2014; Gronke \& Dijkstra 2014). Given what we have learned from the simple models, a complementary route is to continue the investigation with highresolution simulated galaxies. A large ensemble of highresolution simulated galaxies are necessary for a statistical study, and we reserve such an investigation for future work. From the results and analyses we present in this paper, we expect that a more detailed study with simulated galaxies will greatly advance our understanding of the interactions between Ly $\alpha$ emission and CGM/IGM and of galaxy formation through the CGM/IGM environment probed by Ly $\alpha$ emission. 
We thank Renyue Cen and Jordi Miralda-Escudé for useful comments. This work was supported by NSF grant AST-1208891. J.W. was also supported by the Undergraduate Research Opportunities Program (UROP) at the University of Utah. The support and resources from the Center for High Performance Computing at the University of Utah are gratefully acknowledged.

\section{REFERENCES}

Adams, T. F. 1972, ApJ, 174, 439

Ahn, S.-H., Lee, H.-W., \& Lee, H. M. 2000, Journal of Korean Astronomical Society, 33, 29

Ahn, S.-H., Lee, H.-W., \& Lee, H.M. 2001, ApJ, 554, 604

Ahn, S.-H., Lee, H.-W., \& Lee, H.M. 2002, ApJ, 567, 922

Ahn, S.-H., \& Lee, H.-W. 2002, Journal of Korean Astronomical Society, 35,175

Auer, L. H. 1968, ApJ, 153, 783

Avery, L. W., \& House, L. L. 1968, ApJ, 152, 493

Barnes, L. A., Haehnelt, M. G., Tescari, E., \& Viel, M. 2011, MNRAS, 416, 1723

Behrens, C., Dijkstra, M., \& Niemeyer, J. C. 2014, A\&A, 563, A77

Behrens, C., \& Niemeyer, J. 2013, A\&A, 556, A5

Bland, J., \& Tully, B. 1988, Nature, 334, 43

Ciardullo, R., Gronwall, C., Wolf, C., et al. 2012, ApJ, 744, 110

Dekel, A., Birnboim, Y., Engel, G., et al. 2009, Nature, 457, 451

Dijkstra, M., Haiman, Z., \& Spaans, M. 2006, ApJ, 649, 14

Dijkstra, M., Lidz, A., \& Wyithe, J. S. B. 2007, MNRAS, 377, 1175

Gawiser, E., et al. 2007, ApJ, 671, 278

Gronke, M., \& Dijkstra, M. 2014, arXiv:1406.6709

Guaita, L., Gawiser, E., Padilla, N., et al. 2010, ApJ, 714, 255

Harrington, J. P. 1973, MNRAS, 162, 43

Hashimoto, T., Ouchi, M., Shimasaku, K., et al. 2013, ApJ, 765, 70

Hill, G. J., et al. 2008, Astronomical Society of the Pacific Conference Series, 399, 115

Kereš, D., Katz, N., Weinberg, D. H., \& Davé, R. 2005, MNRAS, 363,2

Laursen, P., Sommer-Larsen, J., \& Andersen, A. C. 2009, ApJ, 704,1640
Laursen, P., Sommer-Larsen, J., \& Razoumov, A. O. 2011, ApJ, 728,52

Laursen, P., Duval, F., Östlin, G. 2013, ApJ, 766, 124

Loeb, A., \& Rybicki, G. B. 1999, ApJ, 524, 527

Malhotra, S., \& Rhoads, J. E. 2002, ApJ, 565, L71

McLinden, E. M., Finkelstein, S. L., Rhoads, J. E., et al. 2011, ApJ, 730, 136

McLinden, E. M., Malhotra, S., Rhoads, J. E., et al. 2013, ApJ, 767,48

Neufeld, D. A. 1990, ApJ, 350, 216

Nilsson, K. K., Moeller-Nilsson, O., Moeller, P., Fynbo, J. P. U., \& Shapley, A. E. 2009, MNRAS, 400, 232

Noterdaeme, P., Laursen, P., Petitjean, P., et al. 2012, A\&A, 540, A63

Ouchi, M., et al. 2008, ApJS, 176, 301

Partridge, R.B., \& Peebles, P.J.E. 1967, ApJ, 147, 868

Rhoads, J.E., Dey, A., Malhotra, S., Stern, D., Spinrad, H. Jannuzi, B.T., Dawson, S., Brown, M.J.I., \& Landes, E. 2003, AJ, 125, 1006

Roy, I., Qiu, J.-M., Shu, C.-W., \& Fang, L.-Z. 2009, New Astronomy, 14, 513

Roy, I., Shu, C.-W., \& Fang, L.-Z. 2010, ApJ, 716, 604

Rubin, K. H. R., Prochaska, J. X., Koo, D. C., et al. 2013, arXiv:1307.1476

Shapley, A. E., Steidel, C. C., Pettini, M., \& Adelberger, K. L. 2003, ApJ, 588, 65

Shopbell, P. L., \& Bland-Hawthorn, J. 1998, ApJ, 493, 129

Steidel, C. C., Erb, D. K., Shapley, A. E., et al. 2010, ApJ, 717, 289

Veilleux, S., \& Rupke, D. S. 2002, ApJ, 565, L63

Verhamme, A., Schaerer, D., \& Maselli, A. 2006, A\&A, 460, 397

Verhamme, A., Dubois, Y., Blaizot, J., et al. 2012, A\&A, 546, A111

Weiner, B. J., Coil, A. L., Prochaska, J. X., et al. 2009, ApJ, 692, 187

Wyithe, J. S. B., \& Dijkstra, M. 2011, MNRAS, 415, 3929

Yajima, H., Li, Y., Zhu, Q., et al. 2012, ApJ, 754, 118

Zheng, Z., \& Miralda-Escudé, J. 2002, ApJ, 578, 33

Zheng, Z., Cen, R., Trac, H., \& Miralda-Escudé, J. 2010, ApJ, 716,574

Zheng, Z., Cen, R., Trac, H., \& Miralda-Escudé, J. 2011, ApJ, 726,38

Zheng, Z., Cen, R., Weinberg, D., Trac, H., \& Miralda-Escudé, J. 2011, ApJ, 739, 62 